\title{
Interactive Dissemination: Engaging Stakeholders in the Use of Aggregated Quality Improvement Data for System-Wide Change in Australian Indigenous Primary Health Care
}

\author{
Alison Laycock*, Jodie Bailie, Veronica Matthews and Ross Bailie \\ Menzies School of Health Research, Charles Darwin University, Casuarina, NT, Australia
}

\section{OPEN ACCESS}

Edited by:

Diane Cooper,

University of Western Cape,

South Africa

Reviewed by:

Hanani Tabana,

University of the Western

Cape, South Africa

Godisang Bridget Mookodi, University of Botswana, Botswana

*Correspondence: Alison Laycock

alison.laycock@menzies.edu.au

Specialty section: This article was submitted to Public Health Policy, a section of the journal Frontiers in Public Health

Received: 02 March 2016 Accepted: 18 April 2016 Published: 03 May 2016

Citation: Laycock A, Bailie J, Matthews V and Bailie R (2016) Interactive Dissemination: Engaging

Stakeholders in the Use of Aggregated Quality Improvement Data for System-Wide Change in Australian Indigenous Primary Health Care.

Front. Public Health 4:84. doi: 10.3389/fpubh.2016.00084
Background: Integrating theory when developing complex quality improvement interventions can help to explain clinical and organizational behavior, inform strategy selection, and understand effects. This paper describes a theory-informed interactive dissemination strategy. Using aggregated quality improvement data, the strategy seeks to engage stakeholders in wide-scale data interpretation and knowledge sharing focused on achieving wide-scale improvement in primary health-care quality.

Methods: An iterative process involving diverse stakeholders in Australian Aboriginal and Torres Strait Islander health-care delivery uses aggregated audit data collected across key areas of care. Phases of reporting and online feedback are used to identify: (1) priority areas for improvement; (2) health center, system, and staff attributes that may be important in addressing the identified priority evidence-practice gaps; and (3) strategies that could be introduced or strengthened to enable improvement. A developmental evaluation is being used to refine engagement processes and reports as the project progresses.

Discussion: This innovative dissemination approach is being used to encourage widescale interpretation and use of service performance data by policy-makers, managers, and other stakeholders, and to document knowledge about how to address barriers to achieving change. Through the developmental evaluation, the project provides opportunities to learn about stakeholders' needs in relation to the way data and findings are described and distributed, and elements of the dissemination strategy and report design that impact on the useability and uptake of findings.

Conclusion: The project can contribute to knowledge about how to facilitate interactive wide-scale dissemination and about using data to co-produce knowledge to improve health-care quality.

Keywords: dissemination, knowledge translation, stakeholder engagement, quality, quality of care, primary health care, Indigenous, improvement

Abbreviations: $\mathrm{ABCD}$, audit and best practice for chronic disease; CQI, continuous quality improvement; ESP, engaging stakeholders in identifying priority evidence-practice gaps, barriers and strategies for improvement; PHC, primary health care. 


\section{BACKGROUND}

Integrating theory when developing and evaluating complex quality improvement interventions can help to explain clinical and organizational behavior, inform strategy selection, and understand effects - thereby developing generalizable knowledge $(1,2)$, shortening the time needed to identify conditions required for success and optimizing intervention design (3). Researchers and practitioners need to make explicit the theories used $(3,4)$, as specifying the logic behind continuous quality improvement (CQI) research and practices assists replication and adaptation (5).

Implementation research suggests that by using evidence to identify and link priority gaps in care to theoretical domains that are known to be system enablers or barriers, strategies can be developed that will most likely produce the desired change (6-8). Improvement strategies are more likely to succeed if barriers to effectiveness are identified and addressed at the outset $(9,10)$.

Despite this evidence, there are few examples of how to select and apply theory when developing implementation interventions (11), and limited examples in the literature of how to choose strategies to overcome barriers to implementing care guidelines (12). This paper describes a wide-scale knowledge translation strategy that draws on implementation theory on addressing barriers to improving health care, to implement what we have termed "interactive dissemination."

Dissemination is often linked to implementation of research findings, where interventions aim to reduce or remove barriers and promote change - Hailey and colleagues highlight the challenges of matching research findings to the wider perspectives or requirements of groups being addressed (13). Our "interactive dissemination" strategy is consistent with definitions of dissemination as knowledge transfer and exchange, in which there is interactive exchange between researchers and those they intend to influence and an intention to provide and use information as input to decisions or policies leading to change (13-16). The strategy design synthesizes and translates evidence relevant to the CQI program, supports understanding and use of data, and draws on practical knowledge to identify strategies aligned with implementation settings. These elements are identified as necessary for bridging the "how to" gap between dissemination of evidence and implementation in practice $(17,18)$. Our interactive dissemination strategy, thus, contributes to co-production of knowledge $(19,20)$, which is inherent in our CQI approach within a national research partnership $(21,22)$.

\section{Collaborative Knowledge Production and CQI}

There is recognition of the value of collaborative knowledge production processes through which researchers and service providers share explicit and tacit knowledge to find practical, contextually relevant strategies to improve care quality and health outcomes $(23,24)$. Such processes have potential to help bridge the enduring gap between recommended practice and care delivered $(23,25,26)$. Gaps in care provision that occur across multiple health centers are likely to be due to inadequacies in the broader primary health-care (PHC) delivery system. Improving care quality requires change in approaches that operate at multiple levels of the health system and recognize their interdependencies (27). Stakeholders working at different system levels can help in identifying and addressing inadequacies, sharing knowledge to strengthen systems to achieve wide-scale improvement in care delivery, thereby reducing inequities in health-care access and outcomes between population groups $(21,28,29)$.

Continuous quality improvement activities are widely implemented in PHC. Typically characterized by feedback of systematically collected data, adaptation to local conditions and involvement of participant leaders, they use iterative processes and recognized change methods (e.g., Six-Sigma, Plan-DoStudy-Act cycles) (30). The participatory nature of CQI enables teams to draw on context-specific and experiential knowledge to develop improvement strategies. There is limited understanding of how these CQI principles and processes can be applied at scale to achieve system-wide improvement.

\section{An Interactive Dissemination Strategy Using Aggregated CQI Data}

The dissemination strategy uses CQI data from a program of CQI research and development in Aboriginal and Torres Strait Islander (Australia's Indigenous peoples) PHC in Australia (Box 1). Data contributed over 8 years by 175 health centers delivering care to Indigenous people are aggregated at the national level, and at the Australian state/territory level where sufficient data are available. They comprise clinical audit data on adherence to best practice guidelines representing 56,000 patient records, and data from 492 systems assessments completed by health teams, in priority aspects of PHC. Evidence on this scale enables identification of gaps in care that occur across health centers, and offers a foundation for developing evidence-informed policies and programs to achieve high-level system change and large-scale improvement.

\section{Engaging Stakeholders in the "Identifying Priority Evidence-Practice Gaps, Barriers and Strategies for Improvement (ESP)" Project}

The purpose of the interactive dissemination strategy - the ESP project - is to engage stakeholders working in Indigenous PHC

BOX 1 | The Audit and Best Practice for Chronic Disease (ABCD) National Research Partnership.

In 2010, the ABCD National Research Partnership brought together PHC services, policy, and support organizations and research institutions to guide and support research in improving the quality of Indigenous PHC across Australia (22). Concurrently, the National Centre for Quality Improvement in Indigenous Primary Health Care (www.One21seventy.org.au) was established to provide tools, processes and training to support $\mathrm{CQI}$ and strengthen the implementation of clinical care guidelines. Almost $80 \%$ of health centers using One21seventy services agreed to share their de-identified CQI data for research purposes, forming the most comprehensive and broad-scale dataset relating to health center performance currently available for Indigenous PHC.

Partnership research has highlighted wide variation in performance between different aspects of care and between health centers. While many aspects of care are delivered well in many health centers, there are important gaps between evidence and practice in some aspects of PHC (31-33). 
delivery, management, policy, and research with these aggregated CQI data in order to:

- obtain input in identifying priority evidence-practice gaps, barriers and enablers to addressing the identified priority evidence-practice gaps, and strategies for improvement, and

- encourage use of the data and findings for policy and program development and systems change.

Targeted stakeholders include health practitioners (e.g., doctors, nurses, allied health professionals, Indigenous Health Practitioners), managers and policy-makers working at various levels of the health system, researchers, staff of health service support organizations, and peak bodies representing the interests of Indigenous communities and community-controlled health services.

\section{Context}

Indigenous Australians experience an inequitable burden of illhealth, shorter life expectancy and poorer access to health services compared with the general population $(34,35)$. Contributing factors are complex, relating to colonization and discrimination, social and economic inequalities, and cultural safety. Indigenous Australians access PHC through Indigenous communitycontrolled health and government-managed services designed to meet their needs (36), and through private general practices. Indigenous PHC settings are diverse in geography, governance, and resource provision, and characterized by complex political, cultural, and social interactions.

Continuous quality improvement activities are implemented in many PHC centers that serve Indigenous people, for example, through use of audit and system assessment tools, and Plan-DoStudy-Act approaches. A national CQI framework for Aboriginal and Torres Strait Islander PHC (37) is being established. In this complex health-care environment, it is important to build on strengths and existing knowledge, making optimal use of CQI data and research to help address health inequities.

\section{METHODS}

\section{Theoretical Framework}

The ESP project design is adapted from systematic methods designed to link interventions to modifiable barriers to address evidence-practice gaps. French and colleagues designed a fourstep process comprising guiding questions to identify: (1) an evidence-practice gap, and what needs to be done differently by whom to reduce it; (2) barriers that should be addressed by intervention strategies, based on previously tested theoretical domains relevant to behavior change of health-care professionals $(7,8)$; $(3)$ intervention components that could overcome the barriers and enhance enablers, and; (4) how behavior change can be measured and understood (6). French et al's process has provided the theoretical base for the design of the ESP project, which is guided by the questions: "What are the priority evidence-practice gaps evident in the aggregated CQI data?" "Which barriers and enablers need to be addressed?" "Which strategies could overcome modifiable barriers and enhance enablers?" and "How can we improve dissemination methods to encourage engagement with the data and use of findings?"

\section{Iterative Participatory Approach}

The ESP project uses an iterative and participatory approach. Drawing on action research principles, cycles of systematic enquiry, collaboration, and refinement are applied for the purpose of effecting change (38) and developing theoretical understanding (39).

Separate ESP processes are implemented using audit data collected for child health, chronic illness care, rheumatic heart disease, preventive, maternal, and mental health care. Each process comprises four phases of reporting and stakeholder feedback, culminating in a final report. Each phase comprises a report and linked online survey that uses Likert-scale and openended questions to elicit interpretive and reflective responses. We distribute the reports by email to people in partner organizations and extended networks, encouraging further distribution, discussion, and facilitated group input. The survey tool distinguishes between individual and group responses.

\section{Phase 1}

The first report includes the most recent available CQI data in one aspect of PHC delivery (e.g., child health), aggregated and presented as box and whisker plots with interpretive information and preliminary analysis. This analysis is done by the research team, in collaboration with clinical experts, to identify priorities for improvement. Through the phase 1 survey, we seek feedback on the preliminary priorities, whether they align with respondents' pre-existing perspectives on priorities for improvement and whether other priorities should be included.

\section{Phase 2}

The second report includes the findings from the phase 1 survey (consensus evidence-practice gaps) and trend data over time and by audit cycle for indicators relevant to the identified improvement priorities. We ask respondents to reflect on the trend data and their experience, and answer survey questions to rate potential barriers to improving the priority gaps experienced at different levels of the health system, including system factors relevant to the Indigenous PHC sector (40). Listed domains relating to health center systems, the broader system environment, and staff attributes are drawn from international and national research (7-9, 40, 41). Questions about barriers and enablers relating to individual attributes are informed by the Theoretical Domains Framework $(7,8,42,43)$. Respondents are also asked to rate the accessibility, usability, and usefulness of the report and suggest improvements.

\section{Phase 3}

The third report includes the Phase 2 findings and a summary of published evidence about successful strategies used in CQI, which is intended to stimulate thought and discussion about possible strategies for improving care. We use the Phase 3 survey to find out how stakeholders think existing strategies could be refined, or new strategies developed, to build on system strengths and enablers and overcome the main barriers to addressing the 
priority evidence-practice gaps. Respondents are also asked if the report provides a fair reflection of the main barriers and enablers to improvement in relation to the priority evidence-practice gaps, and how the report could be refined.

\section{Review}

The team incorporates feedback to develop and distribute a draft report of the overall project findings and invites project participants to comment on the representation of findings using a brief online survey. Responses are used to finalize the ESP report in that particular aspect of PHC.

The purpose and elements of ESP phases are summarized in Figure 1.

\section{Concurrent Developmental Evaluation}

A developmental evaluation is being conducted to refine the ESP project structure, materials, and processes as it progresses. One member of our research team (AL) has the lead role on the evaluation, which is expected to contribute to the team's learning and the project's dissemination goals. The evaluation method and resulting project modifications will be described separately.

\section{DISCUSSION}

\section{Use of Aggregated Data for Wide-Scale Quality Improvement}

There is need for innovative dissemination approaches that encourage use of service performance data by policy-makers, managers, practitioners, and community members to identify and address barriers to achieving change. Researchers need to be involved in dialog with these groups to understand policy contexts and how evidence may translate into action (44), and to plausibly link the development of scientifically sound advice with knowledge exchange processes (45). A recent systematic review found timely access to good quality relevant research evidence, collaborations, relationship- and

\begin{tabular}{|c|c|c|c|c|c|}
\hline & $\begin{array}{c}\text { Phase 1 } \\
\text { Priority evidence-practice } \\
\text { gaps }\end{array}$ & $\begin{array}{c}\text { Phase } 2 \\
\text { Barriers/enablers to } \\
\text { improvement }\end{array}$ & \begin{tabular}{|c|} 
Phase 3 \\
Strategies for improvement
\end{tabular} & $\begin{array}{c}\text { Review } \\
\text { Draft final report }\end{array}$ & \\
\hline $\begin{array}{l}\text { Purpose of } \\
\text { phase }\end{array}$ & $\begin{array}{l}\text { To identify priority areas for } \\
\text { improvement (priority } \\
\text { evidence-practice gaps) in } \\
\text { the delivery of Indigenous } \\
\text { PHC }\end{array}$ & $\begin{array}{l}\text { To identify health } \\
\text { centre/system and staff } \\
\text { attributes that may be most } \\
\text { important in addressing the } \\
\text { identified priority evidence- } \\
\text { practice gaps } \\
\text { To confirm phase } 1 \text { findings }\end{array}$ & $\begin{array}{l}\text { To identify new or existing } \\
\text { strategies that could be } \\
\text { introduced or strengthened, } \\
\text { to enable improvement and } \\
\text { address priority evidence- } \\
\text { practice gaps } \\
\text { To confirm phase } 2 \text { findings }\end{array}$ & $\begin{array}{l}\text { To seek feedback on a draft } \\
\text { final report to ensure } \\
\text { accuracy of findings and } \\
\text { messages } \\
\text { To finalise report of ESP } \\
\text { project cycle }\end{array}$ & \\
\hline $\begin{array}{l}\text { Stakeholder } \\
\text { engagement } \\
\text { processes }\end{array}$ & $\begin{array}{l}\text { Input of clinical expert to } \\
\text { assist with preliminary data } \\
\text { analysis } \\
\text { Phase } 1 \text { report sent through } \\
\text { networks, snowballing } \\
\text { Online survey completed by } \\
\text { individuals and groups } \\
\text { Survey data analysed and } \\
\text { findings reported by } \\
\text { research team }\end{array}$ & $\begin{array}{l}\text { Consultation with clinical } \\
\text { expert to assist with trend } \\
\text { data analysis } \\
\text { Phase } 2 \text { report sent to } \\
\text { respondents and through } \\
\text { networks, snowballing } \\
\text { Online survey completed by } \\
\text { individuals and groups } \\
\text { Survey data analysed and } \\
\text { findings reported by } \\
\text { research team }\end{array}$ & $\begin{array}{l}\text { Consultation with clinical } \\
\text { expert to assist with data } \\
\text { analysis } \\
\text { Phase } 3 \text { report sent to } \\
\text { respondents and through } \\
\text { networks, snowballing } \\
\text { Online survey completed by } \\
\text { individuals and groups } \\
\text { Survey data analysed and } \\
\text { findings reported by } \\
\text { research team }\end{array}$ & $\begin{array}{l}\text { Draft final report sent to } \\
\text { respondents and through } \\
\text { networks, snowballing } \\
\text { Online survey completed by } \\
\text { individuals and groups } \\
\text { Overall report and } \\
\text { associated products } \\
\text { finalised by team }\end{array}$ & 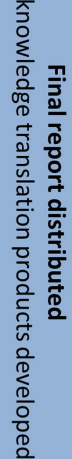 \\
\hline $\begin{array}{l}\text { Information } \\
\text { provided to } \\
\text { participants }\end{array}$ & $\begin{array}{l}\text { Report of aggregated CQI } \\
\text { data about the current } \\
\text { status of care delivery } \\
\text { Preliminary analysis of } \\
\text { priority evidence-practice } \\
\text { gaps }\end{array}$ & $\begin{array}{l}\text { Report on trends over time } \\
\text { for key indicators relevant } \\
\text { to priority evidence- } \\
\text { practice gaps in best } \\
\text { practice care } \\
\text { Phase } 1 \text { findings }\end{array}$ & $\begin{array}{l}\text { Report on key barriers and } \\
\text { enablers identified, relating } \\
\text { to priority evidence- } \\
\text { practice gaps in best } \\
\text { practice care } \\
\text { Evidence brief on barriers, } \\
\text { enablers and strategies for } \\
\text { improving care quality }\end{array}$ & $\begin{array}{l}\text { Draft final report on } \\
\text { findings of all three phases }\end{array}$ & \\
\hline
\end{tabular}

FIGURE 1 | Phases of the ESP project. Note: this process is repeated for each area of care (e.g., child health, chronic illness care). Source: Matthews et al. (26). $\mathrm{PHC}$, primary health care; CQI, continuous quality improvement; ESP, "Engaging Stakeholders in Identifying Priority Evidence-Practice Gaps, Barriers and Strategies for Improvement (ESP)" Project. 
skills-building to be important factors influencing policymakers' use of evidence (46). An Australian review found limited evidence that managers and policy-makers could use to assess the impact of system- and service-level attributes on health outcomes for Indigenous peoples, concluding that more mixed-method research that includes multiple stakeholder perspectives, including those of Indigenous community members, is required (28).

Continuous quality improvement programs typically bring health teams together to plan evidence-informed improvements utilizing clinical data and contextual knowledge to address local evidence-practice gaps in care. In this large-scale project, the challenge of engaging people in "discussion" about care quality based on aggregated data is heightened by limited opportunities for face-to-face or individual-level communication between research team members and stakeholders. Research is needed to determine how CQI processes can be scaled up for higher-level policy and management purposes. It stands to reason that interpretation and use of aggregated CQI data and input by stakeholders in varying roles has potential to identify common and important improvement priorities, and to utilize the collective strengths within PHC services to continue improving health-care quality for Indigenous Australians.

\section{Opportunities for Learning about What Works in Dissemination and Knowledge Co-Production}

Through the developmental evaluation, the team expects to learn more about stakeholders needs and preferences in relation to the way data and findings are described and distributed, and elements of the dissemination strategy and report design that impact on the usability and uptake of findings (47) - including the use of implementation science theory. There is a positive correlation between stakeholder engagement in knowledge production and implementation (23). We hope that by developing understanding of factors that impact on stakeholder participation in the project, and gathering feedback about how to better capture and present stakeholder input, we can contribute knowledge to strengthen the design and impact of knowledge translation processes.

The project should assist in understanding the potential and limitations of online communication to engage health-care stakeholders in wide-scale interactive dissemination processes.

\section{Opportunities for Learning about What Works to Improve PHC Systems and Quality}

The input provided by stakeholders on barriers and enablers, and on strategies for improvement, is valuable in that it reflects tacit knowledge of people working within the health system. We have made innovations to an existing implementation tool used for exploring individual attributes that influence care. Additional questions in the tool are designed to capture knowledge about determinants of performance that operate at health center and system levels $(40,41)$. This exploratory work may inform further studies in health systems and implementation research, including the development of tools to identify barriers to improvement at multiple system levels.

The CQI process used to assess health center systems includes a domain about community linkages (48). A priority for improvement reflected in the aggregated system assessment data is the strengthening of links between health centers and Indigenous communities. Related enablers identified through the ESP process to date include strengthening of community engagement in service delivery design and community leadership for CQI (26). The ESP process has a higher system focus than the health center CQI process; therefore, input from Indigenous peak bodies is important for achieving linkages to influence policy and program design at higher system levels.

The design of wide-scale improvement strategies in the Australian Indigenous PHC context needs to reflect understanding of the holistic nature of Indigenous wellbeing beyond physical health, including healthy connections to culture, community, and land, as well as published evidence and expert knowledge. Findings relating to identified barriers, enablers, and strategies will be reported separately.

Documentation and evaluation of implemented strategies will contribute knowledge about what works and in what contexts to improve PHC for Indigenous communities, and will support adaptation to other settings.

\section{Strengths and Limitations}

A strength of the ESP project is its iterative design using multiple phases. In conjunction with the developmental evaluation, implementing a new dissemination process with each $\mathrm{PHC}$ audit tool dataset provides the team with multiple opportunities to reflect and respond to stakeholder feedback, drawing on evidence and available resources to make and test refinements to processes, reports, and supporting materials. To our knowledge, the level of detail of the data made available from a large number of services across wide geographic scope through this project has not been achieved by other projects.

We are using an open process to engage stakeholders, inviting those who receive reports to distribute them online through their workplaces and networks, and respond to surveys individually or through groups. This strength in the project design puts no limit on the number and diversity of possible participants, thereby enhancing data interpretation and enriching knowledge sharing. We encourage peak bodies representing Indigenous communities to use the reports as a basis for group discussion, enabling further opportunity for community members' input.

The open process also makes it difficult to assess reach and response rates relative to distribution. On balance, the advantages of this snowballing distribution process outweigh the potential limitation in relation to accurate reporting of survey distribution and responses, as a goal of the project is to provide wide-scale access to these CQI data and ESP project findings.

\section{CONCLUSION}

The ESP project uses an innovative theory-informed approach to advance the use of large-scale aggregate CQI datasets, enabling a 
range of stakeholders to identify priority gaps and related barriers in the delivery of best practice PHC in Indigenous communities. Using aggregate CQI data to stimulate discussion among diverse stakeholders on priority evidence-practice gaps in care, and how best to achieve improvement, will contribute knowledge about how to facilitate interactive dissemination and data use.

This process will identify major themes for improving PHCdelivery through changes at the health center and community, regional, and national levels. We expect common themesidentified acrosskey areas of $\mathrm{PHC}$ to be relevant to developing policy and implementing large-scale change to strengthen systems and improve the provision of comprehensive PHC for Indigenous communities across Australia. We anticipate that lessons learned about applying theory to inform the development of improvement interventions, and engaging stakeholders in use of aggregated CQI data for knowledge co-production and system-wide change, will be transferable to other settings.

\section{ETHICS STATEMENT}

Ethical approval for the ABCD National Research Partnership was obtained from research ethics committees in each relevant Australian jurisdiction - Human Research Ethics Committee of the Northern Territory Department of Health and Menzies School of Health Research (HREC EC00153), Central Australian Human Research Ethics Committee (HREC-12-53), New South Wales Greater Western Area Health Service Human Research Committee (HREC/11/GWAHS/23), Queensland Human Research Ethics Committee Darling Downs Health Services District (HREC/11/ QTDD/47), South Australian Aboriginal Health Research Ethics Committee (04-10-319), Curtin University Human Research Ethics Committee (HR140/2008), Western Australian Country Health Services Research Ethics Committee (2011/27), Western Australia Aboriginal Health Information and Ethics Committee (111-8/05), and University of Western Australia Human Research Ethics Committee (RA/4/1/5051). All participants in the ESP Project surveys and evaluation provide individual informed consent.

\section{REFERENCES}

1. Foy R, Øvretveit J, Shekelle PG, Pronovost PJ, Taylor SL, Dy S, et al. The role of theory in research to develop and evaluate the implementation of patient safety practices. BMJ Qual Saf (2015) 20(5):453-9. doi:10.1136/ bmjqs.2010.047993

2. Eccles MP, Grimshaw JM, MacLennan G, Bonetti D, Glidewell L, Pitts NB, et al. Explaining clinical behaviors using multiple theoretical models. Implement Sci (2012) 7(1):99-111. doi:10.1186/1748-5908-7-99

3. Davidoff F, Dixon-Woods M, Leviton L, Michie S. Demystifying theory and its use in improvement. BMJ Qual Saf (2015) 24(3):228-38. doi:10.1136/ bmjqs-2014-003627

4. Davies P, Walker AE, Grimshaw JM. A systematic review of the use of theory in the design of guideline dissemination and implementation strategies and interpretation of the results of rigorous evaluations. Implement Sci (2010) 5:14. doi:10.1186/1748-5908-5-14

5. Kramer DM, Wells RP, Carlan N, Aversa T, Bigelow PP, Dixon SM, et al. Did you have an impact? A theory-based method for planning and evaluating knowledge-transfer and exchange activities in occupational health and safety.

\section{AUTHOR CONTRIBUTIONS}

AL planned and wrote the manuscript. JB provided input and commented on all drafts. VM contributed to conception of the ESP project, led the quantitative analysis of the ABCD data and commented on the draft. RB is the leader of the ABCD National Research Partnership, of which the ESP project is a dissemination strategy. He played a lead role in conceptualization of the ESP process, and contributed to conceptualization and review of the manuscript. All authors read and approved the final manuscript.

\section{ACKNOWLEDGMENTS}

The development of this manuscript was made possible through the active support, enthusiasm, and commitment of members of the ABCD National Research Partnership, especially the participating primary health-care centers that have provided the de-identified data used in the ESP dissemination strategy. The authors gratefully acknowledge Dr. Frances Cunningham, Professor Gillian Harvey, and Dr. Nikki Percival for their comments on the manuscript.

\section{FUNDING}

The ABCD National Research Partnership Project has been supported by funding from the National Health and Medical Research Council (545267) and the Lowitja Institute, and by inkind and financial support from a range of Community Controlled and Government agencies. Alison Laycock is supported by a National Health and Medical Research Council Postgraduate Scholarship (1094595), and by the Centre of Research Excellence: An Innovation Platform for Integrated Quality Improvement in Indigenous Primary Health Care (CRE-IQI, funded by the NHMRC ID 1078927). Ross Bailie's work has been supported by an Australian Research Council Future Fellowship (100100087). The views expressed in this publication are those of the authors and do not necessarily reflect the views of the funding agencies.

Int J Occup Saf Ergon (2013) 19(1):41-62. doi:10.1080/10803548.2013.1107 6965

6. French SD, Green SE, O'Connor DA, McKenzie JE, Francis JJ, Michie S, et al. Developing theory-informed behaviour change interventions to implement evidence into practice: a systematic approach using the Theoretical Domains Framework. Implement Sci (2012) 7:38. doi:10.1186/1748-5908-7-38

7. Huijg J, Gebhardt W, Crone M, Dusseldorp E, Presseau J. Discriminant content validity of a theoretical domains framework questionnaire for use in implementation research. Implement Sci (2014) 9:11. doi:10.1186/1748-5908-9-11

8. Michie S, Johnston M, Abraham C, Lawton R, Parker D, Walker A, et al. Making psychological theory useful for implementing evidence based practice: a consensus approach. Qual Saf Health Care (2005) 14(1):26-33. doi:10.1136/qshc.2004.011155

9. Tugwell P, Robinson V, Grimshaw J, Santesso N. Systematic reviews and knowledge translation. Bull World Health Organ (2006) 84(8):643-51. doi:10.2471/BLT.05.026658

10. Baker R, Camosso-Stefinovic J, Gillies C, Shaw EJ, Cheater F, Flottorp S, et al. Tailored interventions to overcome identified barriers to change: effects on 
professional practice and health care outcomes. Cochrane Database Syst Rev (2010) (3):CD005470. doi:10.1002/14651858.CD005470.pub2

11. Tavender EJ, Bosch M, Gruen RL, Green SE, Michie S, Brennan SE, et al. Developing a targeted, theory-informed implementation intervention using two theoretical frameworks to address health professional and organisational factors: a case study to improve the management of mild traumatic brain injury in the emergency department. Implement Sci (2015) 10(1):74. doi:10.1186/ s13012-015-0264-7

12. Gagliardi AR, Alhabib S, Members of Guidelines International Network Implementation Working Group. Trends in guideline implementation: a scoping systematic review. Implement Sci (2015) 10(1):1-11. doi:10.1186/ s13012-015-0247-8

13. Hailey D, Grimshaw J, Eccles M, Mitton C, Adair CE, McKenzie E, et al. Effective Dissemination of Findings from Research. Edmonton, AB: Institute of Health Economics (2008).

14. Grimshaw JM, Eccles MP, Lavis JN, Hill SJ, Squires JE. Knowledge translation of research findings. Implement Sci (2012) 7:50. doi:10.1186/1748-59087-50

15. National Collaborating Centre for Methods and Tools. Fostering Interactive Exchange and Dissemination [Internet]. McMaster University (2011). Available from: http://www.nccmt.ca/resources/search/79

16. Wilson PM, Petticrew M, Calnan MW, Nazareth I. Disseminating research findings: what should researchers do? A systematic scoping review of conceptual frameworks. Implement Sci (2010) 5:91-106. doi:10.1186/ 1748-5908-5-91

17. Wandersman A, Duffy J, Flaspohler P, Noonan R, Lubell K, Stillman $\mathrm{L}$, et al. Bridging the gap between prevention research and practice: the interactive systems framework for dissemination and implementation. Am JCommunity Psychol (2008) 41(3-4):171-81. doi:10.1007/ s10464-008-9174-z

18. Noonan RK, Wilson KM, Mercer SL. Navigating the road ahead: public health challenges and the interactive systems framework for dissemination and implementation. Am J Community Psychol (2012) 50(3-4):572-80. doi:10.1007/s10464-012-9534-6

19. Heaton J, Day J, Britten N. Collaborative research and the co-production of knowledge for practice: an illustrative case study. Implement Sci (2016) 11:20. doi:10.1186/s13012-016-0383-9

20. Jackson C, Greenhalgh T. Co-creation: a new approach to optimising research impact? Med J Aust (2015) 203(7):2. doi:10.5694/mja15.00219

21. Bailie R, Matthews V, Brands J, Schierhout G. A systems-based partnership learning model for strengthening primary healthcare. Implement Sci (2013) 8:143. doi:10.1186/1748-5908-8-143

22. Bailie R, Si D, Shannon C, Semmens J, Rowley K, Scrimgeour DJ, et al. Study protocol: national research partnership to improve primary health care performance and outcomes for Indigenous peoples. BMC Health Serv Res (2010) 10:129. doi:10.1186/1472-6963-10-129

23. Rycroft-Malone J, Wilkinson J, Burton CR, Harvey G, McCormack B, Graham I, et al. Collaborative action around implementation in Collaborations for Leadership in Applied Health Research and Care: towards a programme theory. J Health Serv Res Policy (2013) 18(3 Suppl):13-26. doi:10.1177/1355819613498859

24. Harvey G. The many meanings of evidence: implications for the translational science agenda in healthcare. Int J Health Policy Manag (2013) 1(3):187-8. doi:10.15171/ijhpm.2013.34

25. Grol R, Grimshaw J. From best evidence to best practice: effective implementation of change in patients' care. Lancet (2003) 362(9391):1225-30. doi:10.1016/S0140-6736(03)14546-1

26. Matthews V, Connors C, Laycock A, Bailie J, Bailie R. Chronic Illness Care for Aboriginal and Torres Strait Islander People: Final Report. ESP Project: Priority Evidence-Practice Gaps and Stakeholder Views on Barriers and Strategies for Improvement. Brisbane: Menzies School of Health Research (2015).

27. Ferlie EB, Shortell SM. Improving the quality of health care in the United Kingdom and the United States: a framework for change. Milbank Q (2001) 79(2):281-315. doi:10.1111/1468-0009.00206

28. Gibson OR, Segal L. Limited evidence to assess the impact of primary health care system or service level attributes on health outcomes of Indigenous people with type 2 diabetes: a systematic review. BMC Health Serv Res (2015) 15:154. doi:10.1186/s12913-015-0803-6
29. De Savigny D, Adam T. Systems Thinking for Health Systems Strengthening. Geneva: World Health Organisation (2009).

30. O’Neill SM, Hempel S, Lim Y-W, Danz MS, Foy R, Suttorp MJ, et al. Identifying continuous quality improvement publications: what makes an improvement intervention 'CQI'? BMJ Qual Saf (2011) 20(12):1011-9. doi:10.1136/ bmjqs.2010.050880

31. Bailie RS, Si D, Connors CM, Kwedza R, O'Donoghue L, Kennedy C, et al. Variation in quality of preventive care for well adults in Indigenous community health centres in Australia. BMC Health Serv Res (2011) 11:139. doi:10.1186/1472-6963-11-139

32. Matthews V, Schierhout G, McBroom J, Connors C, Kennedy C, Kwedza R, et al. Duration of participation in continuous quality improvement: a key factor explaining improved delivery of Type 2 diabetes services. BMC Health Serv Res (2014) 14(1):578. doi:10.1186/s12913-014-0578-1

33. Si D, Bailie R, Dowden M, Kennedy C, Cox R, O’Donoghue L, et al. Assessing quality of diabetes care and its variation in Aboriginal community health centres in Australia. Diabetes Metab Res Rev (2010) 26(6):464-73. doi:10.1002/ dmrr.1062

34. Australian Institute of Health and Welfare. Mortality and Life Expectancy of Indigenous Australians 2008 to 2012. Canberra: AIHW (2014).

35. Australian Health Ministers' Advisory Council. Aboriginal and Torres Strait Islander Health Performance Framework 2014 Report. Canberra: (2015).

36. Baba JT, Brolan CE, Hill PS. Aboriginal medical services cure more than illness: a qualitative study of how Indigenous services address the health impacts of discrimination in Brisbane communities. Int J Equity Health (2014) 13(1):1-10. doi:10.1186/1475-9276-13-56

37. The Lowitja Institute. Final Report - Recommendations for a National CQI Framework for Aboriginal and Torres Strait Islander Primary Health Care. Melbourne: (2014).

38. Green LW, George A, Daniel M, Frankish C, Herbert C, Bowie W, et al. Appendix C: guidelines for participatory research in health promotion. In: Minkler M, Wallerstein N, editors. Community-Based Participatory Research for Health. San Francisco, CA: Jossey-Bass Inc (2003). p. 419-28.

39. Dick B. What can grounded theorists and action researchers learn from each other? In: Bryant A, Charmaz K, editors. The Sage Handbook of Grounded Theory. London: SAGE Publications Ltd (2007). p. 398-416.

40. Schierhout G, Hains J, Si D, Kennedy C, Cox R, Kwedza R, et al. Evaluating the effectiveness of a multifaceted, multilevel continuous quality improvement program in primary health care: developing a realist theory of change. Implement Sci (2013) 8:119. doi:10.1186/1748-5908-8-11

41. Wagner EH, Austin BT, Davis C, Hindmarsh M, Schaefer J, Bonomi A. Improving chronic illness care: translating evidence into action: interventions that encourage people to acquire self-management skills are essential in chronic illness care. Health Aff (2001) 20(6):64-78. doi:10.1377/hlthaff.20.6.64

42. Lipworth W, Taylor N, Braithwaite J. Can the theoretical domains framework account for the implementation of clinical quality interventions? BMC Health Serv Res (2013) 13:530. doi:10.1186/1472-6963-13-530

43. Steinmo SH, Michie S, Fuller C, Stanley S, Stapleton C, Stone SP. Bridging the gap between pragmatic intervention design and theory: using behavioural science tools to modify an existing quality improvement programme to implement "Sepsis Six". Implement Sci (2016) 11:1-12. doi:10.1186/ s13012-016-0376-8

44. Carter N, Lavis JN, MacDonald-Rencz S. Use of modified delphi to plan knowledge translation for decision makers: an application in the field of advanced practice nursing. Policy Polit Nurs Pract (2014) 15(3-4):93-101. doi:10.1177/1527154414544965

45. Contandriopoulos D, Lemire M, Denis J-L, Tremblay É. Knowledge exchange processes in organizations and policy arenas: a narrative systematic review of the literature. Milbank Q (2010) 88(4):444-83. doi:10.1111/j.1468-0009.2010.00608.x

46. Oliver K, Innvar S, Lorenc T, Woodman J, Thomas J. A systematic review of barriers to and facilitators of the use of evidence by policymakers. BMC Health Serv Res (2014) 14:2. doi:10.1186/1472-6963-14-2

47. Grudniewicz A, Bhattacharyya O, McKibbon KA, Straus SE. Redesigning printed educational materials for primary care physicians: design improvements increase usability. Implement Sci (2015) 10(1):1-13. doi:10.1186/ s13012-015-0339-5

48. Si D, Bailie R, Cunningham J, Robinson G, Dowden M, Stewart A, et al. Describing and analysing primary health care system support for chronic 
illness care in Australia's Northern Territory - use of the Chronic Care Model. BMC Health Serv Res (2008) 8:112. doi:10.1186/1472-6963-8-112

Conflict of Interest Statement: The authors declare that the research was conducted in the absence of any commercial or financial relationships that could be construed as a potential conflict of interest.
Copyright $\odot 2016$ Laycock, Bailie, Matthews and Bailie. This is an open-access article distributed under the terms of the Creative Commons Attribution License (CC BY). The use, distribution or reproduction in other forums is permitted, provided the original author(s) or licensor are credited and that the original publication in this journal is cited, in accordance with accepted academic practice. No use, distribution or reproduction is permitted which does not comply with these terms. 